\title{
XI TALLER DE INNOVACIÓN ENFOCADO EN EL DESARROLLO DE LA SOCIEDAD NICARAGÜENSE
}

\author{
Darwin Joel Cajina y Ernesto Gómez Salazar
}

Con el propósito de fortalecer la investigación, innovación, y el desarrollo universitario, docentes y estudiantes de la UNAN - Managua participaron durante la semana del 10 al 16 de mayo en el XI Taller de Innovación, el cual tuvo lugar en el Centro de Convenciones FLAMBOYAN de la Ciudad de Estelí Este evento fue realizado en el marco de cooperación entre la UNAN - Managua y la Universidad de Grand Valley de Estados Unidos.

El taller de innovación consideró como prioridad los ejes de: Salud Pública, Tecnologías de la Comunicación, Medio Ambiente y Cambio Climático. El espacio permitió desarrollar procesos creativos para identificar problemáticas vinculantes al escenario comunitario, a la vez, generar propuestas de proyectos acorde a las necesidades de la sociedad nicaragüense.

El Dr. Paul Lane de la Universidad de Grand Valley de Estados Unidos, organizador del evento, consideró importante el aprendizaje intercultural como oportunidad para el intercambio de ideas, de acuerdo a la realidad social y cultural en que viven las familias de Nicaragua. Esta práctica permitirá establecer relaciones significativas entre docentes y estudiantes con el propósito de para estimular las capacidades de innovación en el seno de lo educativo y comunitario.

\section{Jornada dedicada al Maestro Elmer Cisneros}

Al inicio del taller la MSc. Ramona Rodríguez, Rectora de la UNAN - Managua, dio las palabras de bienvenida a docentes, estudiantes de la UNAN y representantes de la Universidad de Grand Valley y Universidad de Kendall.

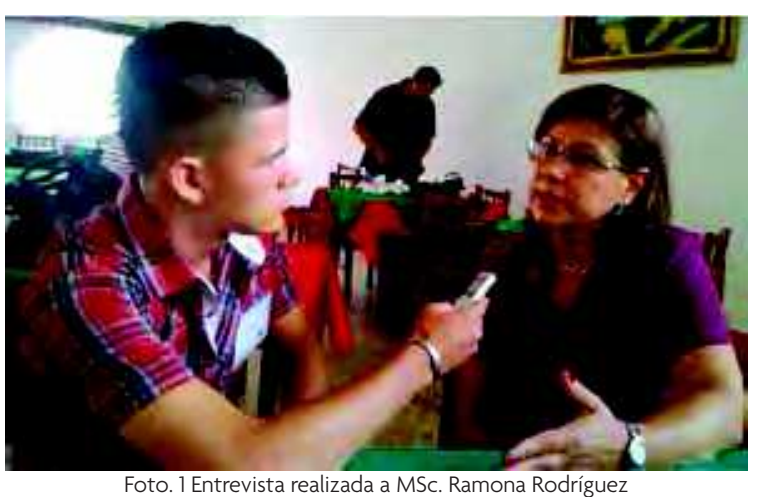
Rectora UNAN - Managua / Foto. Ernesto Gómez S

XI Taller de innovación enfocado en el desarrollo de la sociedad nicaragüense
Durante su intervención, la Maestra Rodríguez dedicó el XI Taller de Innovación a la memoria del Rector Magnífico Elmer Cisneros Moreira (Q e. p. d) quien sin lugar a dudas fue uno de los máximos exponentes del pensamiento "la calidad es el legado", recordó la Rectora de la UNAN - Managua, durante su intervención.

Como muestra de amor, respeto y admiración al Maestro Elmer Cisneros, se dedicó un minuto de silencio a su memoria. Luego el Maestro Máximo Rodríguez,, Decano de FAREM-Estelí, señaló durante su intervención que a lo largo de los años la Universidad ha logrado crear muchos proyectos innovadores en salud, medio ambiente y educación.

\section{Proyectos se tejen con definir el problema}

Los proyectos de innovación toman como punto de partida la cultura de la población. Esto permite un acercamiento directo a los problemas prioritarios para formular de esta manera proyectos con pertinencia y calidad.

El proceso de diseño permitió abordar algunos tips básicos para generar ideas que ayuden a mejorar las condiciones de vida de la población y en particular mejorar la salud pública, tecnología, medio ambiente y cambio climático.

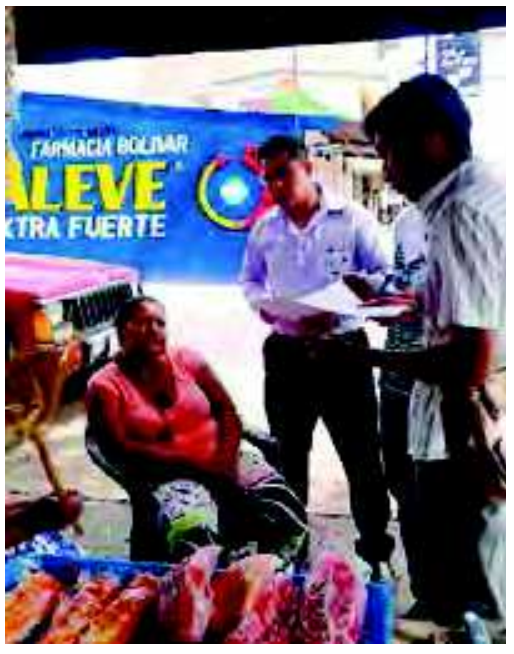

Foto. 2 Trabajo de Campo realizado por los participantes del Taller de Innovación. Foto. Ernesto Gómez Salazar

Darwin Joel Cajina y Ernesto Gómez Salazar 
También se realizó un ejercicio de investigación de mercado, como parte de las principales actividades del taller. La finalidad es definir los niveles de prioridad, actividad que fue posible por medio de las visita casa a casa y negocios de las familias de la ciudad norteña.

Como aspectos de interés la investigación de mercado, se consideraron tres elementos fundamentales: conocer al consumidor, determinar la necesidad e identificar el diseño más apropiado de acuerdo a la demanda.

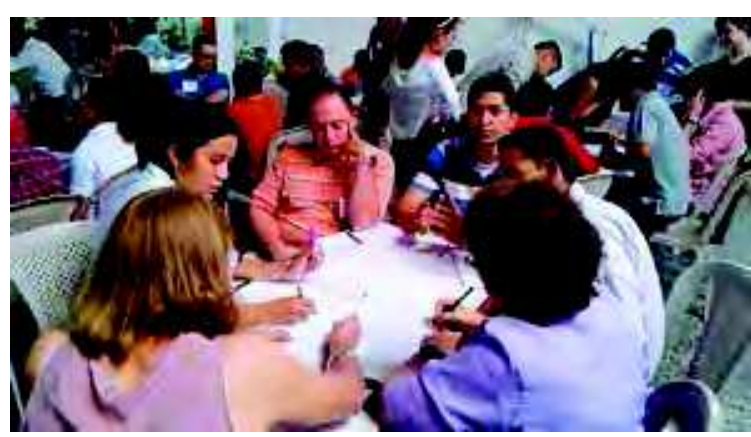

Foto. 3. Participantes analizan en mesas de trabajo la factibilidad de sus productos. Foto. Ernesto Gómez Salazar.

\section{Innovadores despiertan creatividad}

El taller continuó con una serie de actividades enfocadas a la promoción entre los participantes, el espíritu de emprendimiento y la creación de ideas que aporten al desarrollo socioeconómico del país.

Para la dinámica "elaboración del avión de papel", se hizo énfasis en la necesidad de ir más allá del diseño y de las expectativas propuestas.

Cada participante asumió el reto, procediendo de manera creativa a la elaboración de su avión. Acto seguido, los aviones fueron lanzados por los participantes; se seleccionó el que tuviese la capacidad de volar la mayor distancia posible, y que además fuese llamativo y original. Ambos premios fueron ganados por el equipo Azul. El concurso fue dirigido por el profesor Rubén Dolmus.

La formación profesional en el marco del taller avanzó con la ponencia, "El diseño Universal" expuesta por el estudiante Norteamericano, Enrique Andrade. Con su originalidad y energía el expositor indujo a los presentes a despertar sus capacidades de formulación de preguntas con la finalidad de definir un diseño enfocado en las áreas de salud, medio ambiente, cambio climático y tecnología.

\section{Eso no fue todo}

Shelby Owens, estudiante norteamericana, durante su ponencia, trasladó al auditorio al mundo de la antropología y la etnografía en comercio. Estas ciencias estudian al ser humano, utilizando como herramientas metodológicas, para conocer el comportamiento de la cultura, los aspectos sociales y económicos. Esta exposición les permitió a los participantes tener una idea de cómo llegar a los clientes y restantes beneficiados.

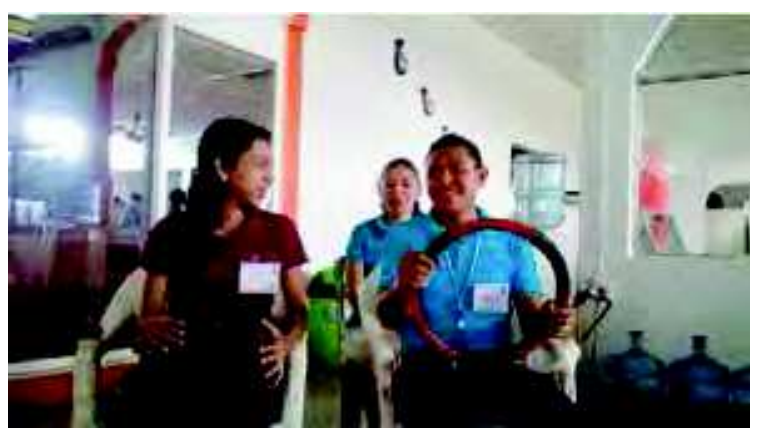

Foto. 4. Simulación del Taxi Pitch, estrategia para llegar al cliente. Foto. Ernesto Gómez Salazar

La exposición "El modelo de negocios de una Organización”, brindada por Rachel Travis, motivó una lluvia de ideas entre los participantes, quienes las estimulados por las experiencias expuestas, comenzaron a externar sus ideas partir de sus experiencias particulares.

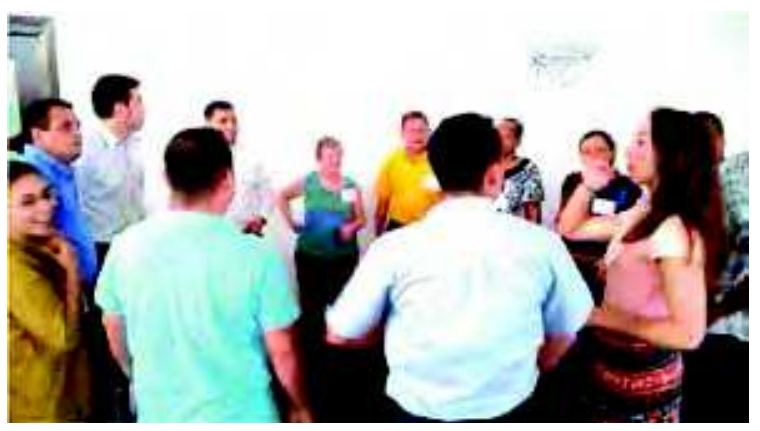

Foto. 5. Actividades de trabajo en equipo desarrolladas durante el taller. Foto. Ernesto Gómez Salazar

Posteriormente, siguiendo la famosa frase; "Hay que unirse, no para estar juntos, sino para hacer algo juntos" de J. Donoso Cortés, los partícipes se trasladaron al trabajo de campo para realizar investigación en las calles del Diamante de las Segovia. El objetivo era definir cada uno de ellos idea acorde a la necesidad más sentida por la población en Estelí.

El Dr. Paul Lane, organizador del Taller, aconsejó a todos los participantes a tener mentes abiertas. Tomar 
en cuenta ideas, identificarse con estas y encontrar soluciones adecuadas que permitan el cambio.

\section{Proyectos deben ser factibles}

Un total de 24 ideas reformadoras y creativas fueron presentadas al plenario el día miércoles 13 de mayo en el Centro de eventos Flamboyán.

Como parte de las actividades del taller se conformaron mesas de trabajo para brindar asesoría en diseño, mercadeo, investigación y clientes con el fin de generar inquietudes en los participantes de cara a reformular sus propuestas. Esto permitió a estudiantes y docentes innovadores abrir sus mentes y de esta manera consolidar sus ideas o bien cambiarlas.

\section{Partir de la idea hasta establecer el negocio}

El ingeniero Ross Ezinga, expuso "La rentabilidad y factibilidad de una idea" con posibilidades de aplicación en Nicaragua. De esta exposición surgieron muchas interrogantes entre los asistentes, como ¿Es rentable mi idea? ¿Es innovadora? ¿Es original? ¿Cumple una función? O ¿Contribuye al futuro?

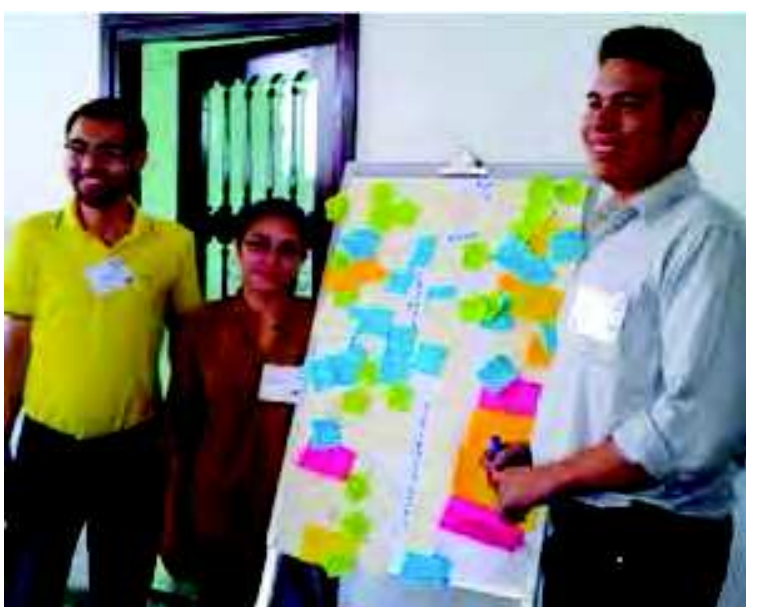

Foto. 6. Análisis de factibilidad de ideas. Foto. Ernesto Gómez Salazar

En cierta medida el criterio del ingeniero Ezinga influyó en los participantes, porque muchas de las ideas primarias seleccionada por ellos fueron modificadas. Pero eso no fue todo, en esta intensa jornada, Mark Becker le dio la sazón económica al proyecto al poner en perspectiva la importancia de tomar en cuenta conceptos como costo y precio. Estos elementos son indispensables indispensables para la aceptación de un producto.

En tanto Kayla Eta, especialista en generación de inventos, explicó que la creación de los modelos y prototipos es uno de los puntos básicos de la innovación. Asimismo afirmó que al crear, nos convertimos en emprendedores al demostrar que una buena idea puede ser concebida como un negocio rentable.

\section{Buenas ideas para la propiedad intelectual}

Las personas con buenas ideas generalmente saben poco o nada de los procesos de fabricación, de tecnología aplicada a las cadenas de producción. Sin embargo los participantes del taller han adquirido características para la promoción del cambio en las nuevas generaciones.

La formación profesional contó con la participación especial durante el taller, del Ministerio de Fomento Industria y Comercio (MIFIC). Javier Collado de representante de esta institución estatal, disertó acerca de la "Propiedad Intelectual enfocada en marcas y patentes".

Motivación y miedo sentido por los Participantes "En esta jornada muchas personas tenían miedo, y se acercaron con temor a enfrentarlo y descubrieron que lo que les aterrorizaba no era tan espantoso como se imaginaban", indicó Sergio Briceño estudiante de Antropología de la Facultad de Humanidades y Ciencias Jurídicas.

Para Michael Raúl Humphrey Toruño, estudiante de Ingeniería en Sistemas de la FAREM-Estelí, "El taller ha superado ampliamente mis expectativas, lo que más me ha gustado es que han salido cosas útiles, cosas que podemos, de alguna manera, aplicar". Agregó que algunas ideas de este taller pueden contribuir a la transformar muchas cosas en el país.

\section{Premiación de los mejores proyectos}

El primer lugar quedó en casa, con el proyecto "Savebag" (Compactador de Basura), el cual consiste en compactar la basura a un menor volumen. Esta propuesta permite el aprovechamiento de la bolsa plástica más tiempo, disminuyendo la contaminación del medio ambiente. El segundo lugar fue para el producto "Estabilizador de Brazo Infantil" y la tercera para un Sistema de recolección inteligente de desechos.

Como un detalle a destacar, el programa de Innovación surgió a partir de una propuesta realizada por la Maestra Ramona Rodríguez en el año 2004, cuando ejercía la función de Decana de la FAREM Estelí.

Desde esa fecha ha pasado once años de trabajo de Darwin Joel Cajina y Ernesto Gómez Salazar 
para posicionar y establecer un espacio dentro de la Universidad.

El trabajo de innovación ha permitido mantener intercambios con el sector público, la iniciativa privada y los movimientos sociales. Además desde el 2010 el Taller se institucionalizó gracias a la gestión del Rector Magnífico Elmer Cisneros.

\section{Retos de la Comisión de innovación de la UNAN - Managua.}

En el marco de este taller, la comisión central de innovación sostuvo una reunión para evaluar los medios materiales y humanos disponibles en los lugares donde trabajan cada uno de los miembros, con el fin de identificar futuras acciones a emprender.

Como resultado de las sesiones de trabajo se establecieron seis estrategias que permitan la promoción y el desarrollo del componente de innovación:

1. Desarrollar réplicas del taller de Innovación AGII 2015

2. Incorporar el modelo de innovación en grado y postgrado

3. Mejorar la formación de docentes y estudiantes sobre procesos de innovación

4. Implementar la Red de Estudiantes Innovadores y Red de Docentes Innovadores
5. Implementar fondos concursables de innovación 6. Desarrollar la red de gestión del conocimiento.

La Comisión central de Innovación desarrollara réplicas del taller de innovación, esto como estrategia que permita la apropiación de la metodología por parte de la UNAN - Managua. Con esta acción se ampliará el segmento de beneficiario del Taller de Innovación.

\section{Facultad de Humanidades y Ciencias Jurídicas ipresente!}

Al taller asistieron los estudiantes: Darwin Cajina de Filología y Comunicación, Sergio Hernández Briceño de Antropología Social, Justo Rivas Rodríguez de Trabajo Social y Nelly Umaña de Psicología.

También participaron los docentes Jonathan Flores del Departamento de Filosofía, Ruth González del Departamento de Historia y Ernesto Gómez Salazar coordinador de la Comisión de Innovación de la Facultad de Humanidades y Ciencias Jurídicas.

Para aquellos estudiantes que deseen formar parte de la Red de estudiantes innovadores o mayor información sobre este tema, contactar a:

MSc. Ernesto Gómez Salazar, correo: ernest.antropology@gmail.com, Teléfono. 2278 - 6764, Ext. 5249 